\title{
Field-line resonance structures in Mercury's multi-ion magnetosphere
}

\author{
Eun-Hwa Kim ${ }^{1}$, Jay R. Johnson ${ }^{1}$, Dong-Hun Lee ${ }^{2,3}$, and Yoo Surn Pyo ${ }^{3}$ \\ ${ }^{1}$ Princeton Plasma Physics Laboratory, Princeton University, NJ 08543, U.S.A. \\ ${ }^{2}$ School of Space Research, Kyung Hee University, Yongin, Gyeonggi 449-701, Republic of Korea \\ ${ }^{3}$ Department of Astronomy and Space Science, Kyung Hee University, Yongin, Gyeonggi 449-701, Republic of Korea
}

(Received April 23, 2012; Revised August 13, 2012; Accepted August 20, 2012; Online published June 10, 2013)

\begin{abstract}
Recently, MESSENGER spacecraft detected transverse waves at Mercury's inner magnetosphere. The magnetic field fluctuations of theses waves are approximately perpendicular to the gradient of magnetic field magnitude which is similar to the field-line resonance characteristics predicted by numerical simulations in two-ion plasmas. In this paper, we perform a wave simulation in a three-ion plasma to consider the effect of multiple heavy ions at Mercury. Because recently observed wave frequencies are near the $\mathrm{He}^{+}, \mathrm{He}^{2+}$ and $\mathrm{H}^{+}$, we adopt multi-ion plasmas that contain $\mathrm{H}^{+}, \mathrm{He}^{2+}$, and $\mathrm{He}^{+}$. The simulation results show that several resonant waves between the ion gyrofrequencies can occur at the same location and also show the modulation of amplitude in time histories. Therefore, the simulation results suggest that it could be possible to observe two or three different resonant frequencies at certain locations in Mercury's magnetosphere.
\end{abstract}

Key words: Mercury, field line resonance, wave absorption, multi-ion effects, ULF waves.

\section{Introduction}

Ultra-low frequency (ULF) waves near the ion gyrofrequencies are often observed by MESSENGER spacecraft inside Mercury's magnetosphere (Boardsen et al., 2009a, b, 2012). Since the Mariner 10 spacecraft detected ULF waves for the first time (Russell, 1989), the identification of ULF waves at Mercury has been a topic of interest. The wave frequency detected by Mariner 10 spacecraft was $0.5 \mathrm{~Hz}$ at $38 \%$ of the proton gyrofrequency $\left(\omega_{\mathrm{cH}}\right)$ and wave frequencies from MESSENGER are near $\omega_{\mathrm{cHe}^{2+}}$ and $\omega_{\mathrm{cH}^{+}}$. Because observations showed that the region around Mercury is filled with heavy ions, such as $\mathrm{Na}^{+}, \mathrm{O}^{+}, \mathrm{K}^{+}$and $\mathrm{He}^{+}$(Zurbuchen et al., 2008, 2011), waves at Mercury require a treatment that includes multiple ions with gyrofrequency effects (Othmer et al., 1999; Glassmeier et al., 2003; Klimushkin et al., 2006; Kim et al., 2008, 2011).

The first observed ULF waves at Mercury were believed to be a field-line resonance (FLR) standing mode along the magnetic field line in single fluid plasma (Russell, 1989). With a field line length of about $4,000 \mathrm{~km}$ based on a dipole model, Russell (1989) estimated that the observed waves could be the fourth harmonic of the fundamental wave. Later, Othmer et al. (1999) suggested that the crossover frequency is the preferred frequency. Kim and Lee (2003) examined the behavior of the Alfvén resonance when $\omega \sim$ $\omega_{\mathrm{cH}}$ and discussed the implications for waves at Mercury in a single-ion plasma. However, Southwood (1997) and Blomberg (1997) both argued that the observed waves cannot be pure standing Alfvén waves because they have a compressional component and polarization aligned in the

Copyright (c) The Society of Geomagnetism and Earth, Planetary and Space Sciences (SGEPSS); The Seismological Society of Japan; The Volcanological Society of Japan; The Geodetic Society of Japan; The Japanese Society for Planetary Sciences; TERRAPUB.

doi:10.5047/eps.2012.08.004 north-radial meridian rather than the east meridian. More recently, Klimushkin et al. (2006) and Kim et al. (2008) suggested that FLRs at Mercury occur at the ion-ion hybrid and/or Alfvén resonance conditions and Kim et al. (2008) showed that the observed waves from the Mariner 10 are not FLRs.

When $\omega \ll \omega_{\mathrm{ce}}, \omega_{\mathrm{pe}}$, where $\omega_{\mathrm{ce}}$ and $\omega_{\mathrm{pe}}$ are the electron gyro- and plasma frequencies, respectively, the basic description of the plasma wave is given by the approximate cold plasma dispersion relation

$$
n_{\perp}^{2} \cong \frac{\left(R-n_{\|}^{2}\right)\left(L-n_{\|}^{2}\right)}{\left(S-n_{\|}^{2}\right)},
$$

where $n_{\|}$and $n_{\perp}$ are refractive indices parallel and perpendicular to the background magnetic field $\left(B_{0}\right)$, respectively. $R, L$ and $S$ are the Stix's tensor elements for two ions (Stix, 1992). This approximate dispersion relation has a resonance when $n_{\perp} \rightarrow \infty$ at

$$
n_{\|}^{2}=S \text {. }
$$

and the cutoffs at

$$
n_{\|}^{2}=R(L) \text {. }
$$

When wave frequencies $\left(\omega_{\mathrm{ii}}\right)$ satisfy the resonance condition $\left(n_{\|}^{2}\left(\omega_{\mathrm{ii}}\right)=S\left(\omega_{\mathrm{ii}}\right)\right)$ in Eq. (2), the ion-ion hybrid (IIH) resonance conditions lie at frequencies between each neighboring ion gyro-frequency pair. In two-fluid plasmas or below the heaviest ion gyrofrequency, this resonance corresponds to the Alfvén resonance $\left(\omega_{\mathrm{A}}\right)$. Therefore, if the plasma contains $m$ ion species, there are $m-1$ ion-ion hybrid resonances and one Alfvén resonance. The wave simulation by Kim et al. (2008) showed that mode-converted waves at the IIH resonance location oscillate linearly, and that the wave energy is strongly guided by $B_{0}$. They also 
(a)

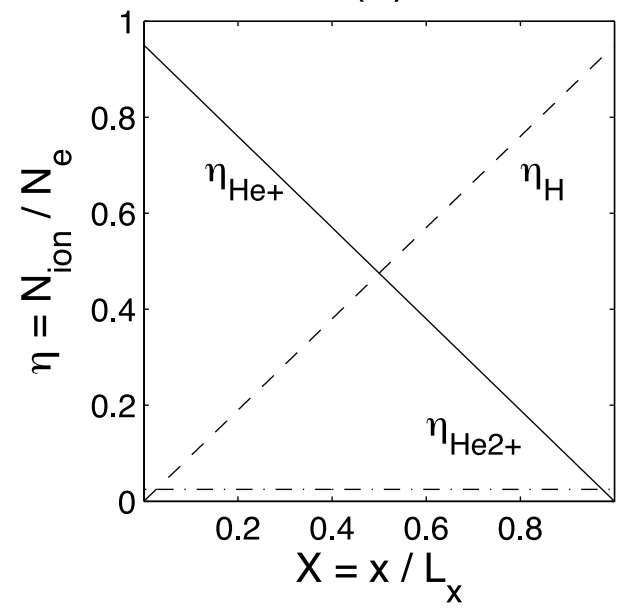

(b)

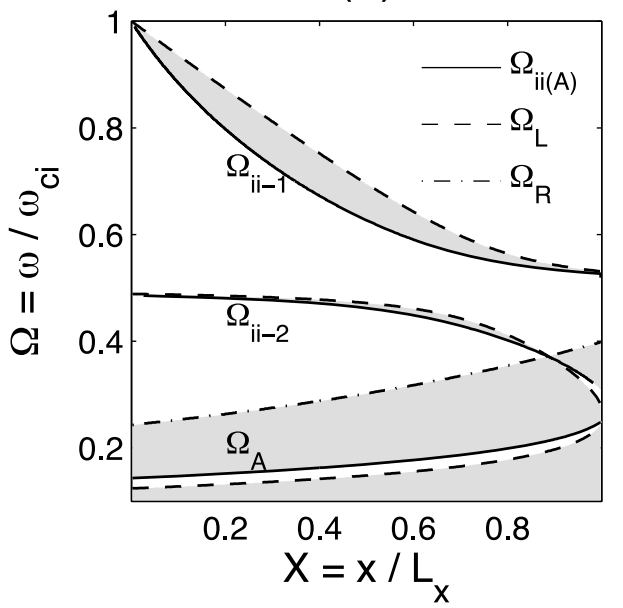

Fig. 1. (a) Ion density ratio ( $\left.\eta=N_{\text {ion }} / N_{\mathrm{e}}\right)$ of $\mathrm{H}^{+}$(dashed), $\mathrm{He}^{+}$(solid), and $\mathrm{He}^{2+}$ (dotted-dashed). Here we assume $\eta_{\mathrm{He} 2}=2.5 \%$. (b) The critical frequencies: cut offs $\Omega_{\mathrm{L}(\mathrm{R})}$ and ion-ion hybrid (and Alfvén) resonance $\left(\Omega_{\mathrm{ii}(\mathrm{A})}\right)$ frequencies. The horizontal and vertical axes are normalized distance $X=x / L_{x}$ and normalized frequency $\Omega=\omega / \omega_{\mathrm{ci}}$. Here shaded regions represent frequency stop-bands where the wave is evanescent.

showed that the coupling is stronger at the IIH resonance compared to the Alfvén resonance. Furthermore, Kim et al. (2011) showed that wave absorption coefficients at the IIH resonance are sensitive to the azimuthal and field-aligned wave numbers as well as heavy ion concentration ratio, thus the FLRs at Mercury can have various radial structures depending on heavy ion density and azimuthal wave numbers.

A recent survey of ULF waves at Mercury showed that the waves are highly transverse at large magnetic latitudes but are more compressional near the equator (Boardsen et al., 2012). They also found that for transverse dominant modes, the magnetic field fluctuations tend to be oriented perpendicular to the estimated field gradient. Previous studies of wave mode conversion at Mercury focused on $\mathrm{Na}^{+}$$\mathrm{H}^{+}$hybrid modes; however the statistical study by Boardsen et al. (2012) shows peaks just below $\omega_{\mathrm{cHe}^{++}}, \omega_{\mathrm{cH}^{+}}$and $2 \omega_{\mathrm{cH}^{++}}$. In order to consider more recent observed ULF waves at Mercury, we perform a wave simulation in a multiion plasma contains $\mathrm{H}^{+}, \mathrm{He}^{2+}$, and $\mathrm{He}^{+}$ions. Our results show that, under the right conditions, the field-aligned waves generated by mode conversion can have wave amplitude beating of several mode-converted waves at the IIH and/or Alfvén resonances.

\section{Numerical Simulation}

We employ the fluid wave simulation model which has been developed by Kim and Lee (2003). Similar to previous wave simulations (Kim et al., 2008), the background magnetic field $B_{0}$ and the electron density $N_{\mathrm{e}}$ are assumed to be constants with $N_{\mathrm{e}}=3 \mathrm{~cm}^{-3}$, and $B_{0}=86 \mathrm{nT}$ at Mercury. The ambient magnetic field, $\mathbf{B}_{0}$, lies in the $z$ direction and the inhomogeneity is introduced in the $x$ direction. Since the Mercury's magnetopause is located near $1.4 \mathrm{R}_{\mathrm{M}}$ (Anderson et al., 2011), we assumed a shorter radial distance of $1 \mathrm{R}_{\mathrm{M}}$ than the magnetopause location in $x$ direction, where $\mathrm{R}_{\mathrm{M}}$ is the Mercury's radius. To save computing time, electron mass is assumed to be $m_{\mathrm{e}}=m_{\mathrm{H}} / 100$. We limit ourselves to harmonic variations in the $y$ and $z$ and all waves are proportional to $\exp \left(i k_{y} y+i k_{z} z\right)$, where $k_{y}$ and $k_{z}$ are the given $y$ and $z$ direction wavenumbers. For simplicity, $k_{y}$ is assumed to be 0 and $k_{z}=2 \pi / L_{z}$, where $L_{z}=1 \mathrm{R}_{\mathrm{M}}$, which is similar to the field line length of $L_{z}=0.93$ at $L=1.5$ in dipole coordinate. The simulation is driven by imposing an impulse in $E_{y}$ at $X=0$ during the interval $0 \leq \tau \leq 2$, where $X=x / L_{x}, \tau=t / t_{\mathrm{ci}}$, and $t_{\mathrm{ci}}=2 \pi / \omega_{\mathrm{ci}}$. The simulation is run from $\tau=0-60$ and the boundaries become perfect reflectors after the impulsive stimulus ends $(\tau=2)$, thus the total energy in the box model will remain constant in time after this interval.

Because $\mathrm{H}^{+}$and $\mathrm{He}^{+}$are major ions at Mercury (Zurbuchen et al., 2008, 2011) and waves near the $\mathrm{He}^{2+}$ gyrofrequency are observed (Boardsen et al., 2009a, b), we adopt an electron- $\mathrm{H}^{+}-\mathrm{He}^{2+}-\mathrm{He}^{+}$plasma and the electron density is also assumed to be sum of the ion densities. For simplicity, the $\mathrm{He}^{2+}$ density ratio to electron density $\left(\eta_{\mathrm{He} 2}=N_{\mathrm{He} 2} / N_{\mathrm{e}}\right)$ is assumed to be 0.025 , however, in order to see the multi-ion effects easily, we assume decreasing $\mathrm{He}^{+}$density ratio in space. Figure 1(a) shows the ion density ratio to electron density $\left(\eta_{\text {ion }}=N_{\text {ion }} / N_{\mathrm{e}}\right)$ profile. We assumed the $\mathrm{H}^{+}$density ratio $\left(\eta_{\mathrm{H}}\right)$ increases from 0 at $X=0$ to 0.95 at $X=1$ while $\mathrm{He}^{+}$density $\left(\eta_{\mathrm{He}}\right)$ decreases from 0.95 to 0 . Using this profile, we calculate the normalized critical frequencies to $\omega_{\mathrm{ci}}\left(\Omega=\omega / \omega_{\mathrm{ci}}\right)$, such as cutoffs $\Omega_{\mathrm{L}(\mathrm{R})}$ (where $n_{\|}^{2}=L(R)$ ) and ion-ion hybrid (and Alfvén) resonances $\left(\Omega_{\mathrm{ii}}\right.$ and $\left.\Omega_{\mathrm{A}}\right)$ frequencies. These frequencies are plotted in Fig. 1(b) and shaded regions represent frequency stop-bands where waves are evanescent. Different from the two-ion case predicted by Kim et al. (2008), the IIH resonance modes in between the proton and heaviest ion gyrofrequencies split into the two branches of $\Omega_{\mathrm{ii}-1}$ (where $\Omega_{\mathrm{cHe}^{2+}}<\Omega_{\mathrm{ii}-1}<\Omega_{\mathrm{cH}}$ ) and $\Omega_{\mathrm{ii}-2}$ (where $\Omega_{\mathrm{cHe}^{+}}<\Omega_{\mathrm{ii}-2}<\Omega_{\mathrm{cHe}^{2+}}$ ). Here, $\Omega_{\mathrm{ii}-1}$ decreases from 1 to 0.527 and $\Omega_{\mathrm{ii}-2}$ decreases from 0.486 to 0.312 . When the impulsive input is excited at $X=0$, most waves in these resonance frequency ranges encounter the resonance location without cutoffs. In this case, the maximum absorption can increase up to $100 \%$ (Lee et al., 2008; Kim et al., 2011). However, below $\Omega_{\mathrm{cHe}^{2+}}$ most waves except between 
(a) $B_{x}$

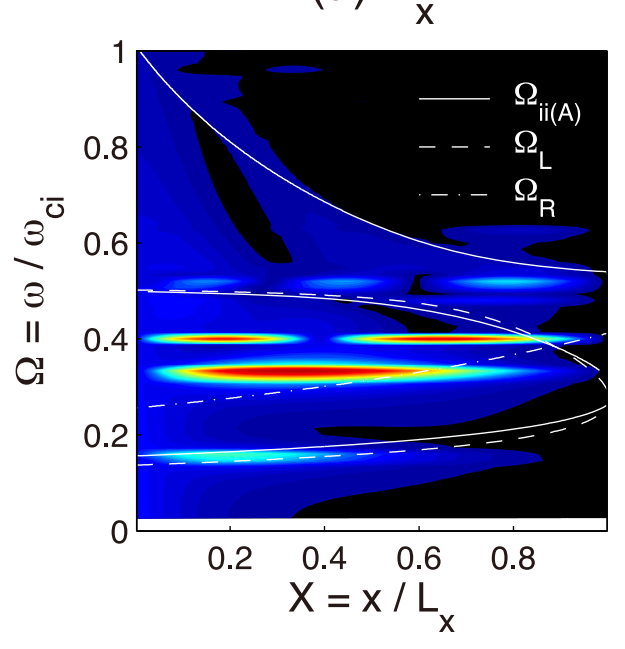

(b) $B$

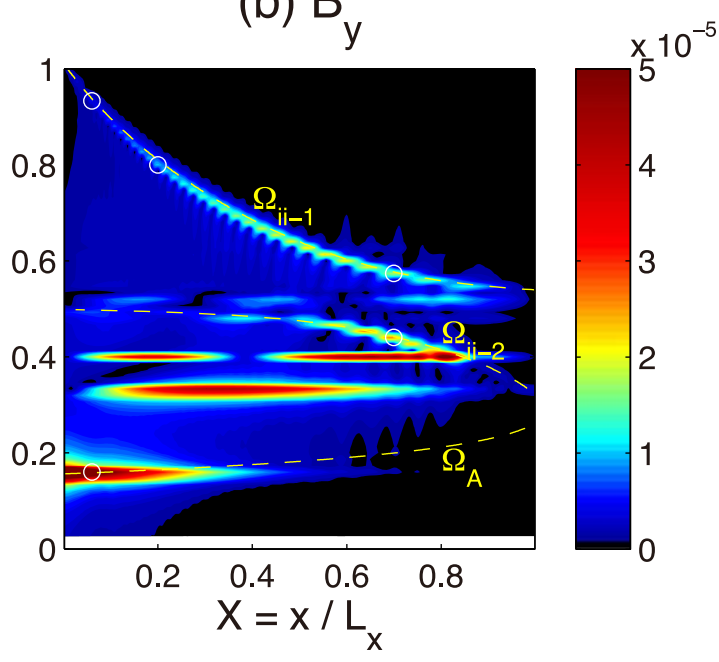

Fig. 2. The wave spectra of the perturbed electric field: (a) the radial component of $B_{x}$ and (b) the azimuthal component of $B_{y}$. The horizontal axis is the direction of inhomogeneity, $X$, and the vertical axis is a normalized frequency that ranges from 0 to 1 . The white solid, dashed, and dashed-dotted

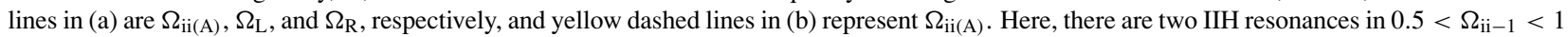
and $0.25<\Omega_{\mathrm{ii}-2}<0.5$, and one Alfvén resonance in $\Omega_{\mathrm{A}}<0.25$. The open circles mark the point chosen for time histories.

the local cutoff of $\Omega_{\mathrm{R}}=0.124$ and resonance frequency of $\Omega=0.143$ at $X=0$ cannot propagate toward the resonances. Therefore, if the wave power is not enough large to penetrate the wave stop gap, the mode conversion at the Alfvén resonance does not occur.

We store time histories of the electromagnetic fields at each grid point in $X$ during the simulation running time of $0<\tau<60$ and obtain the wave power spectra through the fast Fourier transform. Figure 2 shows the wave spectra of (a) $B_{x}$ (radial component), which is and (b) $B_{y}$ (azimuthal component).

In this figure, harmonics of the global cavity modes appear in both components near $\Omega=0.14$ which is in between $\Omega_{\mathrm{L}}<\Omega<\Omega_{\mathrm{A}}$, and $\Omega=0.32,0.38$, and 0.51 which are larger than the local $\Omega_{\mathrm{R}}$. In contrast with the global cavity wave modes, the continuous spectrum appears only in azimuthal component of $B_{y}$. For $\Omega>\Omega_{\mathrm{He}^{+}}$, there are two continuous bands whose frequencies decrease with increasing $X$. These continuous spectrum are impulsively excited by the broadband compressional source at $X=0$ and corresponds to the IIH resonant conditions of $\Omega_{\mathrm{ii}-1}$ and $\Omega_{\mathrm{ii}-2}$. For $\Omega<\Omega_{\mathrm{He}^{+}}$, power enhancement of $B_{y}$ only occurs at $\Omega=0.14$ near $X=0$. This mode is associated with Alfvén resonance. Different to $\Omega_{\mathrm{ii}-1}$ and $\Omega_{\mathrm{ii}-2}$, most waves in the frequency range of $\Omega_{\mathrm{A}}$ at $X=0$ cannot propagate into the simulation domain as shown in Fig. 1(b) but some wave energy can directly reach $\Omega_{\mathrm{A}}$ near $X=0$.

Figure 2 also shows that each resonant wave mode branches have different power at different locations. Resonant waves at $\Omega_{\mathrm{ii}-1}$ have strong power in wide range $0 \leq X \leq 0.95$, while waves at $\Omega_{\mathrm{ii}-2}$ are strong for $0.6 \leq X \leq 0.85$ but weak for $0 \leq X<0.6$. Moreover, the Alfvén resonance mode $\left(\Omega_{\mathrm{A}}\right)$ occurs only near $X \approx 0$. Therefore, single or multiple frequencies of modeconverted wave can be detected at certain locations. For instance, two mode-converted waves can be detected at $X=0.06\left(\Omega_{\text {high }}=\Omega_{\text {ii-1 }} \approx 0.93\right.$ and $\left.\Omega_{\text {low }}=\Omega_{\mathrm{A}} \approx 0.16\right)$ and $X=0.7\left(\Omega_{\text {high }}=\Omega_{\text {ii- } 1} \approx 0.57\right.$ and $\Omega_{\text {low }}=\Omega_{\text {ii- } 2} \approx$ $0.44)$ while a single frequency of mode-converted wave $\left(\Omega_{\text {high }}=\Omega_{\text {ii-1 }} \approx 0.8\right.$ ) can be observed at $X=0.2$ (these frequencies are marked as open circles in Fig. 2(b)). In order to examine the coupling properties, we plot the time histories of $B_{x}$ and $B_{y}$ in Fig. 3, which are obtained using the inverse Fourier transforms. In this figure, the first and second panels are wave time histories of higher $\left(\Omega_{\text {high }}\right)$ and lower frequencies $\left(\Omega_{\text {low }}\right)$ of magnetic azimuthal component $\left(B_{y}\right)$ at (a) $X=0.06$, (b) 0.2 , and (c) 0.7 , respectively. We also plot the wave time histories of the azimuthal $\left(B_{y}\right)$ and radial $\left(B_{x}\right)$ components in the third and fourth panels of Fig. 3 that show superposition of two resonant waves of $\Omega_{\text {high }}$ and $\Omega_{\text {low }}$. However, because only one resonant wave mode is dominant at $X=0.2$, the second panel is remained as blank.

Similar to the previous multi-ion simulation study by Kim et al. (2008), wave azimuthal components with single frequency grow in time as shown in the first and second panels of Fig. 3. When mode-converted waves contain two different frequencies, waves can show modulation of the amplitude in time. For $X=0.06$, because frequency ratio of two resonant waves $\Omega_{\text {high }} / \Omega_{\text {low }}$ is 6.27 and the amplitude of $\Omega_{\text {low }}$ is greater than $\Omega_{\text {high }}$, wave amplitude beating is not significantly appeared in the third panel. Different to case of $X=0.06$, for $X=0.7$ in Fig. 3(c), $\Omega_{\text {high }} / \Omega_{\text {low }}=1.31$ and the wave amplitudes are similar for both frequencies, therefore, the amplitude beating in time is clearly shown in the third panel of Fig. 3(c). In this case, when two wave amplitudes are assumed to be the same and constant in time, the beat period $T_{\text {beat }}$ can be approximated to be $1 /(\Delta \Omega) \approx$ 7.7 , which is consistent with the simulation results. In contrast to the increase of the azimuthal component $\left(B_{y}\right)$, the radial component $\left(B_{x}\right)$ damps for two mode-converted waves (fourth panels in Figs. 3(a) and (c)) and for a single mode (Fig. 3(b)). When there are two mode-converted waves appeared at the same location in Fig. 3(a) and (c), $B_{x}$ 
(a) $X=0.06$

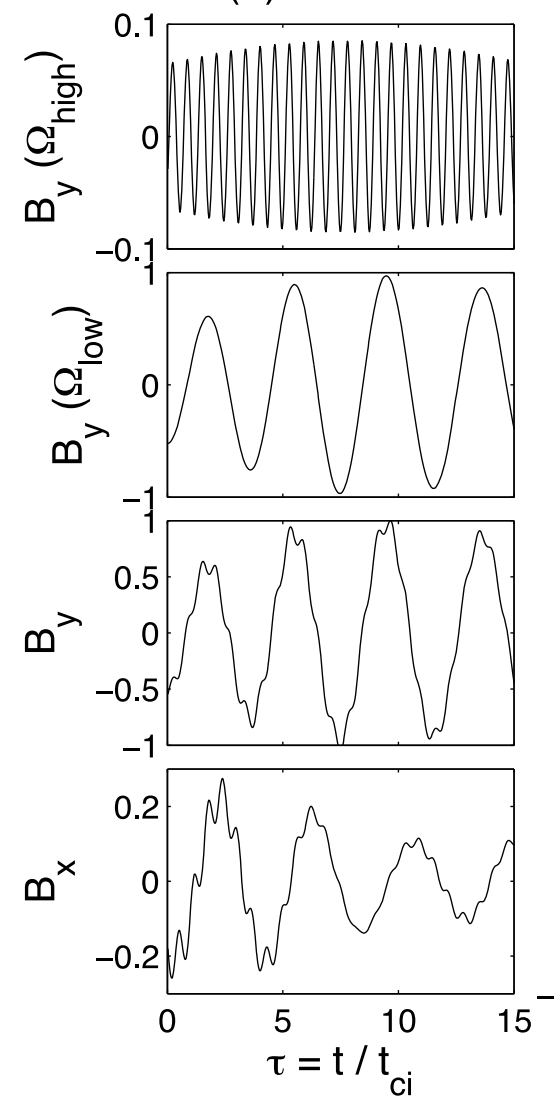

(b) $X=0.2$
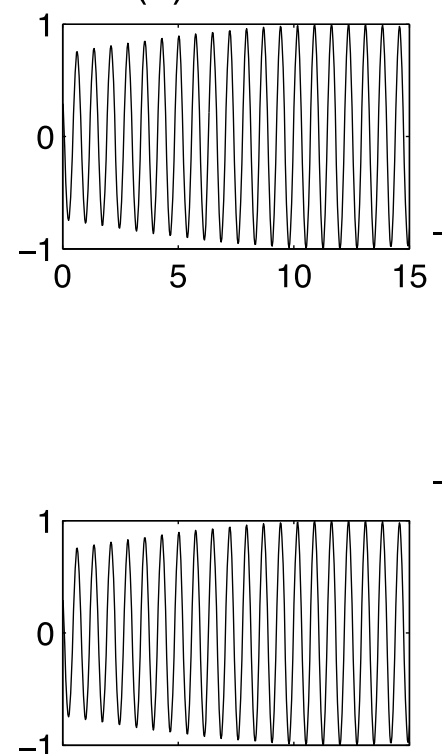

0.2

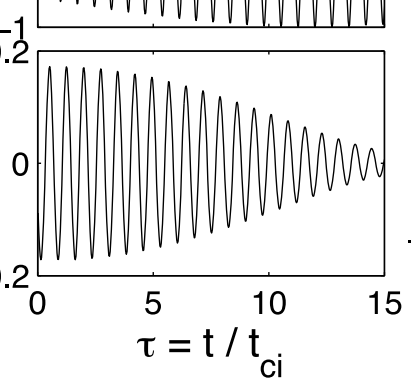

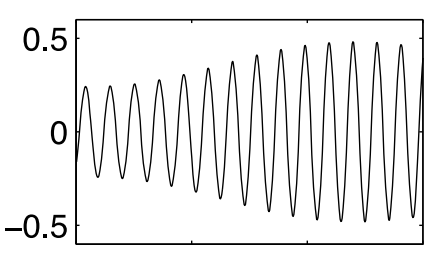

(c) $X=0.7$
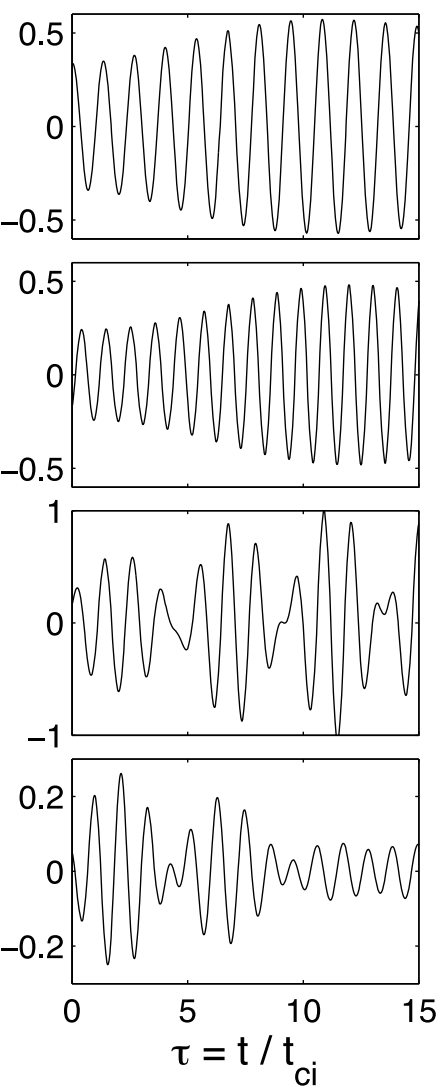

Fig. 3. Time histories of electric fields at the IIH resonance marked as circles at (a) $X=0.06$, (b) $X=0.2$, and (c) $X=0.7$. The first and second rows are time histories of the azimuthal component for $\Omega_{\text {high }}$ and $\Omega_{\text {low }}$, respectively. Here, for $\Omega_{\text {high }}=0.8$ at $X=0.2$, for $\Omega_{\text {high }}=0.93$ and $\Omega_{\text {low }}=0.16$ at $X=0.06$, and for $\Omega_{\text {high }}=0.57$ and $\Omega_{\text {low }}=0.44$ at $X=0.7$, respectively. The third and fourth rows are wave modulations of azimuthal and radial components.

also show wave amplitude beating with damping in time.

\section{Discussion and Summary}

In this paper, we perform a wave simulation in three-ion plasmas to consider multiple heavy ion effects at Mercury. The simulation results show that several resonant waves between the ion gyrofrequencies can occur at certain locations and show wave amplitude beating in time histories.

Mercury's magnetosphere contains heavier (e.g., $\mathrm{O}^{+}$, $\mathrm{K}^{+}, \mathrm{Na}^{+}$) and lighter ions (e.g., $\mathrm{He}^{+}, \mathrm{He}^{2+}$ ) and MESSENGER spacecraft have recently detected ULF wave events near $\Omega_{\mathrm{He}^{+}}$and $\Omega_{\mathrm{H}^{+}}$(Boardsen et al., 2009a, b, 2012). The observed magnetic component of transverse waves tends to be strongly linearly polarized and oriented approximately perpendicular to the estimated field gradient (which is plasma inhomogeneity). This is consistent with wave simulation results: In Figs. 2 and 3, the $B_{y}$ component which is perpendicular to the plasma inhomogeneity is dominant at the mode-converted waves. Previous twoion simulations demonstrated the IIH resonance occurs in $\mathrm{H}^{+}-\mathrm{Na}^{+}$plasmas but did not include $\mathrm{He}^{+}$or $\mathrm{He}^{2+}$ ions. In this paper, we show how small amount $\mathrm{He}^{2+}$ change the FLRs structure. The simulation results suggest that several resonant waves between the ion gyrofrequencies can occur at a certain location and show wave amplitude beating in time histories. Therefore, it could possible to observe two or three different resonant frequencies in Mercury's magnetosphere. In addition, because the IIH resonance is the result of ion-ion effects and those frequencies are affected only by the relative ion density ratio rather than the absolute number density, the wave frequency could be used to estimate the local heavy ion density if a spacecraft detects multiple resonances (Kim et al., 2008).

In this study, a cold plasma model is adopted. However, because of the small dimensions of Mercury's magnetosphere (for instance, magnetopause is located near 1.4 $\mathrm{R}_{\mathrm{M}} \sim 3400 \mathrm{~km}$ and the large gyro radii of ions $\sim 100$ $1000 \mathrm{~km}$, kinetic effects can play an important role for the generation and propagation of ULF waves (Glassmeier et al., 2004; Glassmeier and Espley, 2006; Slavin et al., 2009). When the kinetic effects are included, the compressional wave can be converted into the short wavelength ion Bernstein and/or ion cyclotron waves at the IIH resonance. These mode converted waves can be strongly damped in the vicinity of the mode-conversion surface via electron Landau damping or damped by ions at the Doppler broadened ion cyclotron resonances (e.g., Phillips et al., 1995; Intrator et al., 1986; Jaeger et al., 2003, 2006). Therefore, although the cold plasma wave simulation in this study shows the resonant absorption characteristics near the IIH and Alfvén resonance clearly, more detailed investigations on mode-converted waves including kinetic effects at Mer- 
cury's multi-ion plasmas are necessary.

Acknowledgments. The work at the Princeton University was supported by NASA grants (NNH09AM53I, NNH09AK63I, and NNH11AQ46I), NSF grant ATM0902730, and DOE contract DEAC02-09CH11466. The work at the Kyung Hee University was supported by KRF-2008-31-C00375 and the WCU program through NRF funded by MEST of Korea (R31-10016). Eun-Hwa Kim thanks to Peter Damiano for useful discussions.

\section{References}

Anderson, B. J. et al., The global magnetic field of mercury from MESSENGER orbital observations, Science, 333, 1859-1862, doi:10.1126/science.1211001, 2011.

Boardsen, S. A., B. J. Anderson, M. H. Acuña, J. A. Slavin, H. Korth, and S. C. Solomon, Narrow-band ultra-low-frequency wave observations by MESSENGER during its January 2008 flyby through Mercury's magnetosphere, Geophys. Res. Lett., 36, L01104, doi:10.1029/2008GL036034, 2009a.

Boardsen, S. A., J. A. Slavin, B. J. Anderson, H. Korth, and S. C. Solomon, Comparison of ultra-low-frequency waves at Mercury under northward and southward IMF, Geophys. Res. Lett., 36, L18106, doi:10.1029/2009GL039525, 2009b.

Boardsen, S. A., J. A. Slavin, B. J. Anderson, H. Korth, D. Schriver, and S. C. Solomon, Survey of coherent $\sim 1 \mathrm{~Hz}$ waves in Mercury's inner magnetosphere from MESSENGER observations, J. Geophys. Res., 117, A00M05, doi:10.1029/2012JA017822, 2012.

Blomberg, L. G., Mercury's magnetosphere, exosphere and surface: Lowfrequency field and wave measurements as a diagnostic tool, Planet. Space Sci., 45, 143-148, 1997.

Glassmeier, K.-H., P. N. Mager, and D. Y. Klimushkin, Concerning ULF pulsations in Mercury's magnetosphere, Geophys. Res. Lett., 30, 1928, doi:10.1029/2003GL017175, 2003.

Glassmeier, K.-H., D. Klimushkin, C. Othmer, and P. Mager, ULF waves at Mercury: Earth, the giants, and their little brother compared, Adv. Space Res., 33, 1875-1883, doi:10.1016/j.asr.2003.04.047, 2004.

Glassmeier, K.-H. and J. Espley, ULF waves in planetary magnetospheres, in Magnetospheric ULF Waves: Synthesis and New Directions, edited by K. Takahashi et al., pp. 341-349, AGU, 2006.

Intrator, T. et al., Alfvén ion-ion hybrid wave heating in the Phaedrus-T tokamak, Phys. Plasmas, 3, 1331-1339, doi:10.1063/1.871786, 1986.

Jaeger, E. F. et al., Sheared poloidal flow driven by mode conversion in Tokamak plasmas, Phys. Rev. Lett., 90(19), 195001,
doi:10.1103/PhysRevLett.90.195001, 2003.

Jaeger, E. F. et al., Global-wave solutions with self-consistent velocity distributions in ion cyclotron heated plasmas, Nucl. Fusion, 46(7), S397S408, doi:10.1088/0029-5515/46/7/S02, 2006.

Kim, E.-H. and D.-H. Lee, Resonant absorption of ULF waves near the ion cyclotron frequency: A simulation study, Geophys. Res. Lett., 30(24), 2240, doi:10.1029/2003GL017918, 2003.

Kim, E.-H., J. R. Johnson, and D.-H. Lee, Resonant absorption of ULF waves at Mercury's magnetosphere, J. Geophys. Res., 113, A11207, doi:10.1029/2008JA013310, 2008.

Kim, E.-H., J. R. Johnson, and K.-D. Lee, ULF wave absorption at Mercury, Geophys. Res. Lett., 38, L16111, doi:10.1029/2011GL048621, 2011.

Klimushkin, D. Y., P. N. Mager, and K.-H. Glassmeier, Axisymmetric Alfvén resonances in a multi-component plasma at finite ion gyrofrequency, Ann. Geophys., 24, 1077-1084, 2006.

Lee, D.-H., J. R. Johnson, K. Kim, and K.-S. Kim, Effects of heavy ions on ULF wave resonances near the equatorial region, J. Geophys. Res., 113, A11212, doi:10.1029/2008JA013088, 2008.

Othmer, C., K.-H. Glassmeier, and R. Cramm, Concerning field line resonances in Mercury's magnetosphere, J. Geophys. Res., 104(A5), 10,369-10,378, doi:10.1029/1999JA900009, 1999.

Phillips, C. K. et al., Ion cyclotron range of frequencies heating and current drive in deuterium, tritium plasmas, Phys. Plasmas, 2(6), 2427, doi:10.1063/1.871266, 1995.

Russell, C. T., ULF waves in the Mercury magnetosphere, Geophys. Res. Lett., 16, 1253-1256, 1989.

Slavin, J. A. et al., MESSENGER observations of magnetic reconnection in Mercury's magnetosphere, Science, 324, 606, 2009.

Southwood, D. J., The magnetic field of Mercury, Planet. Space Sci., 45, 113-117, 1997.

Stix, N., Waves in Plasmas, American Institute of Physics, New York, 1992.

Zurbuchen, T. H. et al., MESSENGER observations of the composition of Mercury's ionized exosphere and plasma environment, Science, 321, 90-92, doi:10.1126/science.1159314, 2008.

Zurbuchen, T. H. et al., MESSENGER observations of the spatial distribution of planetary ions near Mercury, Science, 333, 1862-1865, doi:10.1126/science.1211302, 2011.

E.-H. Kim (e-mail: ehkim@pppl.gov), J. R. Johnson, D.-H. Lee, and Y. S. Pyo 